\title{
Twisted identities in Coxeter groups
}

\author{
Axel Hultman
}

Received: 7 March 2007 / Accepted: 2 November 2007 / Published online: 17 November 2007

(C) Springer Science+Business Media, LLC 2007

Abstract Given a Coxeter system $(W, S)$ equipped with an involutive automorphism $\theta$, the set of twisted identities is

$$
\iota(\theta)=\left\{\theta\left(w^{-1}\right) w \mid w \in W\right\} .
$$

We point out how $\iota(\theta)$ shows up in several contexts and prove that if there is no $s \in S$ such that $s \theta(s)$ is of odd order greater than 1 , then the Bruhat order on $\iota(\theta)$ is a graded poset with rank function $\rho$ given by halving the Coxeter length. Under the same condition, it is shown that the order complexes of the open intervals either are PL spheres or $\mathbb{Z}$-acyclic. In the general case, contractibility is shown for certain classes of intervals. Furthermore, we demonstrate that sometimes these posets are not graded.

For the Poincaré series of $\iota(\theta)$, i.e. its generating function with respect to $\rho$, a factorisation phenomenon is discussed.

Keywords Coxeter groups · Bruhat order · Twisted identities · Twisted involutions

\section{Introduction}

Let $(W, S)$ be a Coxeter system with an involutive automorphism $\theta$. A twisted identity is an element of the form $\theta\left(w^{-1}\right) w$ for $w \in W$. In other words, the set $\iota(\theta)$ of twisted identities is the orbit of the identity element under the twisted conjugation action of $W$ on itself. Our terminology appeared in [9] and stems from the fact that when $\theta=\mathrm{id}$, the only twisted identity is the identity element $e$.

\footnotetext{
A. Hultman $(\varangle)$

Department of Mathematics, KTH, 10044 Stockholm, Sweden

e-mail: axel@math.kth.se
} 
As will be demonstrated in Section 3, the study of $\iota(\theta)$ is motivated by its appearance in a variety of situations. For example, certain orbit decompositions of symmetric varieties have a close relationship with the subposet $\operatorname{Br}(\iota(\theta))$ of the Bruhat order on $W$ which is induced by $\iota(\theta)[13,14]$. Also, the Bruhat order on $W$ itself appears as a special case of $\operatorname{Br}(\iota(\theta))$; see Example 3.2.

This article is chiefly devoted to $\operatorname{Br}(\iota(\theta))$. The questions that we strive to answer emerge from a context which we now briefly describe.

A twisted involution in $W$ is an element which is sent to its inverse by $\theta$. Denote by $\mathfrak{I}(\theta)$ the set of twisted involutions. Clearly,

$$
\iota(\theta) \subseteq \Im(\theta) \subseteq W .
$$

Let $\operatorname{Br}(X)$ denote the subposet of the Bruhat order on $W$ induced by $X \subseteq W$. It is a fact that $\operatorname{Br}(W)$ is graded with rank function given by the Coxeter length $\ell$. Furthermore, a fundamental result due to Björner and Wachs [3] asserts that (the order complex of) every (open) interval in $\operatorname{Br}(W)$ is a $\mathrm{PL}$ sphere. Recent results on $\operatorname{Br}(\mathfrak{I}(\theta))$ produce a similar picture. It is graded with rank function $\rho=\left(\ell+\ell^{\theta}\right) / 2$, where $\ell^{\theta}$ is the twisted absolute length [9]. Moreover, every interval in $\operatorname{Br}(\Im(\theta))$ is a PL sphere [10].

In the spirit of the above description, we pose the following problems:

1. For which $(W, S)$ and $\theta$ is $\operatorname{Br}(\iota(\theta))$ a graded poset?

2. Describe the topology of the intervals of $\operatorname{Br}(\iota(\theta))$.

We do not know the complete solution to either of the problems. Our main results on $\operatorname{Br}(\iota(\theta))$ are these partial answers:

- In Theorem 4.6 it is shown that if $s \theta(s)$ never is of odd order for $s \in S$ unless $s$ is a fixed point of $\theta$, then $\operatorname{Br}(\iota(\theta))$ is graded with rank function $\rho$. For example, this is always the case if $W$ is of odd rank and its Coxeter graph is a tree. By way of contrast, there exist $(W, S)$ and $\theta$ such that $\operatorname{Br}(\iota(\theta))$ is not graded; see Example 4.7.

- Under the same conditions on $s \theta(s)$ as above, every interval in $\operatorname{Br}(\iota(\theta))$ is either $\mathbb{Z}$-acyclic (i.e. has trivial reduced integral homology) or a PL sphere. The latter case occurs precisely when the interval coincides with an interval in $\operatorname{Br}(\mathfrak{I}(\theta))$. This is Theorem 4.12. Dropping these conditions, the homotopy type of an interval can be computed in certain special cases (Theorems 4.8 and 4.9).

In addition to the above results, we also investigate what we call the Poincaré series of $\iota(\theta)$. This is the rank generating function of $\operatorname{Br}(\iota(\theta))$ whenever it is graded. Specifically, we provide a simple necessary condition for an intriguing factorisation phenomenon to occur and demonstrate that this condition also is sufficient in the context of finite Coxeter groups. The general case is left open.

The structure of the remainder of the paper is as follows. In the next section we gather preliminaries on poset topology and Coxeter groups, including some new material on twisted involutions, that we need in the sequel. In Section 3 we then give an account of various natural contexts where $\iota(\theta)$ and $\operatorname{Br}(\iota(\theta))$ appear. After that, we turn to the study of $\operatorname{Br}(\iota(\theta))$ in Section 4. Section 5 is concerned with Poincaré 
series considerations. Finally, we mention several open problems and give further comments in Section 6.

\section{Preliminaries}

\subsection{Posets and combinatorial topology}

Say that a poset is locally finite if every interval is finite. A locally finite poset equipped with a minimum element is graded if in any given interval all maximal chains have the same cardinality.

With any finite poset $P$, we associate the order complex $\Delta(P)$. It is the (abstract) simplicial complex whose simplices are the chains in $P$. Whenever we make topological statements about $P$, we have the corresponding properties of $\Delta(P)$ in mind. We make no notational distinction between an abstract simplicial complex and its geometric realisation.

Given a regular CW complex $\Delta$, we define the face poset $P(\Delta)$ to be the set cells in $\Delta$ ordered by inclusion. By convention, we include the empty cell as the minimum element in $P(\Delta)$.

A simplicial complex is a $P L$ (piecewise linear) ball if it has a common subdivision with a simplex. Similarly, it is a $P L$ sphere if it has a common subdivision with the boundary of a simplex. In particular, a PL sphere is of course homeomorphic to a sphere (and similarly for balls).

We now review elements of Forman's discrete Morse theory [7]. Its formulation in terms of matchings, to which we adhere, is due to Chari [4]. For unexplained terminology from combinatorial topology as well as further background, we refer to [1].

Let $\Delta$ be a finite regular CW complex. A matching on the face poset $P(\Delta)$ is an involution $M: Q \rightarrow Q$, for some $Q \subseteq P(\Delta)$ such that for all $q \in Q$, either $M(q) \prec q$ or $M(q) \succ q$, where $\prec$ is the covering relation in $P(\Delta)$. In other words, $M$ is nothing but a graph-theoretic matching of the Hasse diagram of $P(\Delta)$. The unmatched elements, i.e. the members of $P(\Delta) \backslash Q$, are called the critical cells.

The matching $M$ is acyclic if whenever we have a sequence in $Q$ of the form

$$
q_{0} \prec M\left(q_{0}\right) \succ q_{1} \prec M\left(q_{1}\right) \succ \cdots \prec M\left(q_{t-1}\right) \succ q_{t}
$$

with $q_{1} \neq q_{0}$, it holds that $q_{t} \neq q_{0}$. A nice way to interpret this condition is as follows. If, in the Hasse diagram of $\Delta(P)$, we direct the matching edges upwards and the others downwards, then this directed graph is acyclic if and only if $M$ is an acyclic matching.

Without loss of generality, we will always assume that the empty cell is not critical, i.e. that $Q$ includes the minimum element ${ }^{1}$.

Our acyclic matching $M$ determines a way to collapse $\Delta$ onto a (possibly nonregular) critical complex $\Delta^{M}$ consisting of the critical cells together with the vertex which was matched with the empty cell. In the process, incidences among the cells may change. In some situations, however, this is not a problem.

\footnotetext{
${ }^{1}$ Otherwise, we may just extend $M$ to include it. There is always a matching partner for the empty cell available, because no acyclic matching can match all 0-dimensional cells with 1-dimensional ones.
} 
Theorem 2.1 (Forman [7]) If $M$ is an acyclic matching on $P(\Delta)$ which is complete (i.e. has no critical cells), then $\Delta$ is collapsible, in particular contractible.

Theorem 2.1 has the following consequence, which happens to be well-suited for some of our applications.

Corollary 2.2 Suppose $M$ is an acyclic matching on $P(\Delta)$ with set of critical cells $C$. If $C$ is an order filter (i.e. $c \in C, b \geq c \Rightarrow b \in C$ ), then $\Delta$ is homotopy equivalent to the complex $\Delta^{M}$ obtained from $\Delta$ by collapsing the complex of non-critical cells to a point.

Proof A standard result in topology asserts that for a $\mathrm{CW}$ complex $A$ with contractible subcomplex $A_{0}$, the quotient map $A \rightarrow A / A_{0}$ is a homotopy equivalence. Since $C$ is an order filter, $\Delta \backslash C$ is a subcomplex of $\Delta$. This subcomplex is contractible by Theorem 2.1 .

\subsection{Coxeter groups}

The reader is assumed to be familiar with the basics of Coxeter group theory. Here, bits and pieces are reviewed in order to agree on notation. We refer to the textbooks [2] and [11] for further details.

Henceforth, let $(W, S)$ be a Coxeter system with $|S|<\infty$. The Coxeter length function is $\ell: W \rightarrow \mathbb{N}$. If $w=s_{1} \cdots s_{k} \in W$ and $\ell(w)=k$, the word $s_{1} \cdots s_{k}$ is called a reduced expression for $w$. Here, and in what follows, symbols of the form $s_{i}$ are always elements in $S$. We make no notational distinction between a word in the free monoid over $S$ and the element in $W$ which it represents; we rely on the context to clarify our intentions.

Given Coxeter generators $s, s^{\prime} \in S$, we let $m\left(s, s^{\prime}\right)$ denote the (possibly infinite) order of $s s^{\prime}$. This information is gathered in the Coxeter graph with vertex set $S$ and an edge labelled $m\left(s, s^{\prime}\right)$ connecting $s$ and $s^{\prime}$ if and only if they do not commute (i.e. if and only if $m\left(s, s^{\prime}\right) \geq 3$ ). As is customary, omitted edge labels are understood to equal 3.

The set of reflections in $W$ is $T=\left\{w^{-1} s w \mid s \in S\right\}$. The absolute length $\ell^{\prime}(w)$ is the minimal $k$ such that $w=t_{1} \cdots t_{k}$ for some $t_{i} \in T$.

Given $w \in W$, we define its (right) descent set by

$$
D_{R}(w)=\{s \in S \mid \ell(w s)<\ell(w)\} .
$$

For $J \subseteq S$, let $W_{J}$ be the standard parabolic subgroup of $W$ generated by $J$. If $W_{J}$ is finite, it has a longest element which is denoted by $w_{J}$. In $W_{J}$, it is characterised by the fact that $D_{R}\left(w_{J}\right)=J$. Following tradition, we write $w_{0}=w_{S}$.

We now define the Bruhat order. Among several equivalent definitions, the one which follows is probably best suited for our purposes. A disadvantage is that it is not obvious that what it defines is indeed a partial order.

Definition 2.3 The Bruhat order on $W$ is the partial order defined by $u \leq v$ if and only if for every reduced expression $s_{1} \cdots s_{k}$ for $v$ there exist $1 \leq i_{1}<\overline{i_{2}}<\cdots<$ $i_{m} \leq k$ such that $u=s_{i_{1}} \cdots s_{i_{m}}$. 


\subsection{Twisted involutions in Coxeter groups}

By an automorphism of $(W, S)$ we mean a group automorphism of $W$ which preserves $S$. Such an automorphism is determined by the corresponding automorphism of the Coxeter graph.

Let $\theta$ be an involutive automorphism of $(W, S)$. Recall from the introduction that the set of twisted involutions in $W$ is

$$
\Im(\theta)=\left\{w \in W \mid \theta(w)=w^{-1}\right\} .
$$

Observe that $\mathfrak{I}(\mathrm{id})$ is the set of ordinary involutions.

The combinatorics of $\mathfrak{I}(\theta)$ can be described in terms of "reduced expressions" in a way which is remarkably similar to that of $W$ itself. We now proceed to review the parts of this theory that we will use in the sequel. Most of this appeared in [10], but some results are new.

Define a set of symbols $\underline{S}=\{\underline{s} \mid s \in S\}$. There is an action of the free monoid $\underline{S}^{*}$ on the set $W$ defined by

$$
w \underline{s}= \begin{cases}w s & \text { if } \theta(s) w s=w \\ \theta(s) w s & \text { otherwise }\end{cases}
$$

and $w \underline{s}_{1} \cdots \underline{s}_{k}=\left(\cdots\left(\left(w \underline{s}_{1}\right) \underline{s}_{2}\right) \cdots\right) \underline{s}_{k}$. Abusing notation, we will write $\underline{s}_{1} \cdots \underline{s}_{k}$ for $e \underline{s}_{1} \cdots \underline{s}_{k}$, where $e \in W$ is the identity. The relevance of all this is the following:

Proposition 2.4 (Proposition 3.5 in [10]) The orbit of e under the $\underline{S}^{*}$-action is $\mathfrak{I}(\theta)$, i.e. the twisted involutions are the elements of the form $\underline{s}_{1} \cdots \underline{s}_{k}$.

For $w \in \mathfrak{I}(\theta)$, the $\operatorname{rank} \rho(w)$ is the smallest $k$ such that $w=\underline{s}_{1} \cdots \underline{s}_{k}$ for some $s_{i} \in S$. Then, the expression $\underline{s}_{1} \cdots \underline{s}_{k}$ is called a reduced $\underline{S}$-expression for $w$.

It follows from [9, Theorem 4.8] that $\operatorname{Br}(\mathfrak{I}(\theta))$, the Bruhat order on twisted involutions, is graded with rank function $\rho$. More precisely, it was shown that the rank function is $\left(\ell+\ell^{\theta}\right) / 2$, where $\ell^{\theta}$ is the twisted absolute length, but it follows from the proof that this function coincides with $\rho$. The twisted absolute length was defined differently in [9], but here is a description which is more convenient for our purposes:

Proposition 2.5 Suppose $\underline{s}_{1} \cdots \underline{s}_{k}$ is a reduced $\underline{S}$-expression for $w \in \mathfrak{I}(\theta)$. Then, $\ell^{\theta}(w)$ is the number of indices $i \in[k]=\{1, \ldots, k\}$ that satisfy $\underline{s}_{1} \cdots \underline{s}_{i-1} \underline{s}_{i}=$ $\underline{s}_{1} \cdots \underline{s}_{i-1} s_{i}$.

Proof Let $\lambda(w)$ be the asserted number (a priori, it depends on the choice of reduced $\underline{S}$-expression). One can check that $\ell(\theta(s) w s)=\ell(w) \Leftrightarrow \theta(s) w s=w$ for all $w \in \mathfrak{I}(\theta)$ and $s \in S$ (see [10, Lemma 3.4]). Furthermore, by [10, Lemma 3.8], $\rho(w \underline{s})>\rho(w) \Leftrightarrow \ell(w s)>\ell(w)$. The construction of the $\underline{S}^{*}$-action therefore implies

$$
\ell(w)=2 \rho(w)-\lambda(w) .
$$

Thus, $\lambda+\ell=2 \rho=\ell^{\theta}+\ell$. 
As the terminology suggests, $\ell^{\mathrm{id}}=\ell^{\prime}$; see [9].

Either by a simple induction argument based on Proposition 2.5 or as an immediate consequence of [9, Definition 4.5], we conclude that the twisted identities are the twisted involutions of vanishing twisted absolute length:

$$
\iota(\theta)=\left\{w \in \mathfrak{I}(\theta) \mid \ell^{\theta}(w)=0\right\}=\{w \in \mathfrak{I}(\theta) \mid \ell(w)=2 \rho(w)\} .
$$

A useful consequence is that $w \underline{s} \in \iota(\theta)$ whenever $w \in \iota(\theta)$ and $s \in D_{R}(w)$.

Twisted involutions have reduced $\underline{S}$-expressions of a convenient form, as shown by the next lemma which is due to Springer [17].

Lemma 2.6 (Proposition 3.3(a) in [17]) Any $u \in \mathfrak{I}(\theta)$ with $\ell^{\theta}(u)=k$ has a reduced $\underline{S}$-expression of the form $\underline{s}_{1} \cdots \underline{s}_{t}$ with the property that for some $0 \leq i \leq t, \underline{s}_{1} \cdots \underline{s}_{i}$ $i$ a reduced $\underline{S}$-expression for the longest element $w_{J}$ in a $\theta$-invariant standard parabolic subgroup $W_{J}, J \subseteq S$, with $\ell^{\theta}\left(w_{J}\right)=k$.

The following fundamental lemma will be put to use repeatedly throughout the paper. It is completely analogous to the corresponding property in $W$ (due to Deodhar [5, Theorem 1.1]) and explains why $\operatorname{Br}(\mathfrak{I}(\theta))$ and $\operatorname{Br}(W)$ are so similarly behaved. The first two parts are [10, Lemma 3.9]. Together, they imply the third.

Lemma 2.7 (Lifting property) Let $s \in S$ and $u, w \in \mathfrak{I}(\theta)$ with $u \leq w$. Suppose $s \in D_{R}(w)$. Then,

(i) $u \underline{s} \leq w$.

(ii) $s \in D_{R}(u) \Rightarrow u \underline{s} \leq w \underline{s}$.

(iii) $s \notin D_{R}(u) \Rightarrow u \leq w \underline{s}$.

Next, we show that $\operatorname{Br}(\Im(\theta))$, like $\operatorname{Br}(W)$, can be described in terms of subwords. The result extends Richardson and Springer's [13, Corollary 8.10] ${ }^{2}$ from finite to general Coxeter groups (and adjusts it to our definition of the $\underline{S}^{*}$-action which differs somewhat from the one given in [13]).

Theorem 2.8 (Subword property for $\mathfrak{I}(\theta)$ ) Let $u, v \in \mathfrak{I}(\theta)$ be given. Then, $u \leq v$ in the Bruhat order if and only if for every reduced $\underline{S}$-expression $\underline{s}_{1} \cdots \underline{s}_{k}$ for $v$ there exist $1 \leq i_{1}<i_{2}<\cdots<i_{m} \leq k$ such that $u=\underline{s}_{i_{1}} \cdots \underline{s}_{i_{m}}$.

Proof We begin with the "only if" direction, so suppose $u \leq v$ and choose a reduced $\underline{S}$-expression $\underline{s}_{1} \cdots \underline{s}_{k}$ for $v$. If $s_{k} \notin D_{R}(u)$, then the lifting property shows that $u \leq$ $v \underline{s}_{k}=\underline{s}_{1} \cdots \underline{s}_{k-1}$. By induction on the rank of $v$, we are done. Otherwise, if $s_{k} \in$ $D_{R}(u)$, we have $u \underline{s}_{k} \leq v \underline{s}_{k}$. It follows, again by induction, that $u \underline{s}_{k}=\underline{s}_{i_{1}} \cdots \underline{s}_{i_{m}}$ for some $1 \leq i_{1}<i_{2}<\cdots<i_{m} \leq k-1$. Acting by $\underline{s}_{k}$ yields the desired result.

Now consider the "if" part of the assertion. Assume $\underline{s}_{1} \cdots \underline{s}_{k}$ is a reduced $\underline{S}$-expression for $v$ and let $u=\underline{s}_{i_{1}} \cdots \underline{s}_{i_{m}}$ for some $1 \leq i_{1}<i_{2}<\cdots<i_{m} \leq k$.

\footnotetext{
${ }^{2}$ More precisely, [13, Corollary 8.10] applies to a partial order on $\Im(\theta)$ which later was shown to coincide with $\operatorname{Br}(\Im(\theta))$ in [14].
} 
Given $t \in[k]$, let $v_{t}=\underline{s}_{1} \cdots \underline{s}_{t}$ and $u_{t}=\underline{s}_{i_{1}} \cdots \underline{s}_{i_{l}}$, where $i_{l} \leq t<i_{l+1}$ (with $i_{0}=1$, $\left.i_{m+1}=k+1\right)$. Assuming by induction that $u_{t-1} \leq v_{t-1}$, we either have $u_{t}=u_{t-1} \leq$ $v_{t-1}<v_{t-1} \underline{s}_{t}=v_{t}$ or (by the lifting property) $u_{t}=u_{t-1} s_{t} \leq v_{t-1} \underline{s}_{t}=v_{t}$. Thus, $u=$ $u_{k} \leq v_{k}=v$.

\section{Manifestations of $\iota(\theta)$}

Henceforth, let $(W, S)$ be a finitely generated Coxeter system with an involutive automorphism $\theta$. Recall that $\theta$ is determined by an automorphism of the Coxeter graph.

It should be clear by now that the goal of this paper is to study certain properties of $\iota(\theta)$. In this section, we motivate this task by indicating some situations in which $\iota(\theta)$ naturally shows up.

Example 3.1 Consider a connected reductive linear algebraic group $G$ (over $\mathbb{C}$, say). Let $B$ be a Borel subgroup and $T \subseteq B$ a maximal torus. Given an involutive automorphism $\Theta: G \rightarrow G$ which preserves $T$ and $B$, let $K$ be the fixed point subgroup. By means of left translations, $B$ acts on the symmetric variety $G / K$. This gives rise to a finite number of orbits that may be ordered by containment of their Zariski closures. Richardson and Springer [13, 14] studied this poset $V$ by defining an order preserving map $\varphi: V \rightarrow \operatorname{Br}(\Im(\theta))$. Here, the underlying Coxeter group $W$ is the Weyl group $N / T$ (where $N$ is the normaliser of $T$ in $G$ ) and $\theta$ is induced by $\Theta$.

In general, $\varphi$ is neither injective nor surjective. However, $\iota(\theta)$ is always contained in the image. Moreover, $\varphi$ produces an isomorphism $V \cong \operatorname{Br}(\iota(\theta))$ in certain interesting cases. For instance, with $G=\mathrm{SL}_{2 n}$, we may define $\Theta$ so that $K \cong \mathrm{Sp}_{2 n}$ as in [13, Example 10.4]. The corresponding poset $V$, governing the orbit decomposition of $\mathrm{SL}_{2 n} / \mathrm{Sp}_{2 n}$, is then isomorphic to $\operatorname{Br}(\iota(\theta))$, where $W$ is the symmetric group $\mathfrak{S}_{2 n}$ and $\theta$ is given by conjugation with the longest element in $W$ (i.e. the reverse permutation $i \mapsto 2 n+1-i$ ). Figure 1 displays this poset for $n=3$.

Example 3.2 (cf. Example 10.1 in [13] and Example 3.2 in [10]) Suppose $\theta$ is the involution $\theta: W \times W \rightarrow W \times W$ given by $(v, w) \mapsto(w, v)$, where $W$ is any Coxeter group. Observe that $\iota(\theta)=\left\{\left(w, w^{-1}\right) \mid w \in W\right\}$. Hence, we have a bijection $W \leftrightarrow \iota(\theta)$ in this case. Furthermore, it is clear from Definition 2.3 that this bijection gives a poset isomorphism $\operatorname{Br}(\iota(\theta)) \cong \operatorname{Br}(W)$. Therefore, (Bruhat orders on) twisted identities generalise (Bruhat orders on) Coxeter groups.

Example 3.3 Let $\operatorname{Fix}(\theta)$ denote the fixed point subgroup of $\theta$. It is known [8, 12, 19] that $\operatorname{Fix}(\theta)$ itself is a Coxeter group with the following canonical set of Coxeter generators:

$$
\left\{w_{J} \mid J=\{s, \theta(s)\}, m(s, \theta(s))<\infty, s \in S\right\} .
$$

Observe that $\theta\left(w^{-1}\right) w=\theta\left(v^{-1}\right) v \Leftrightarrow v w^{-1} \in \operatorname{Fix}(\theta) \Leftrightarrow v \in \operatorname{Fix}(\theta) w$. In other words, there is a bijection between $\iota(\theta)$ and the set of cosets $\operatorname{Fix}(\theta) \backslash W$. Thus, $\iota(\theta)$ can be thought of as a quotient of Coxeter groups. 


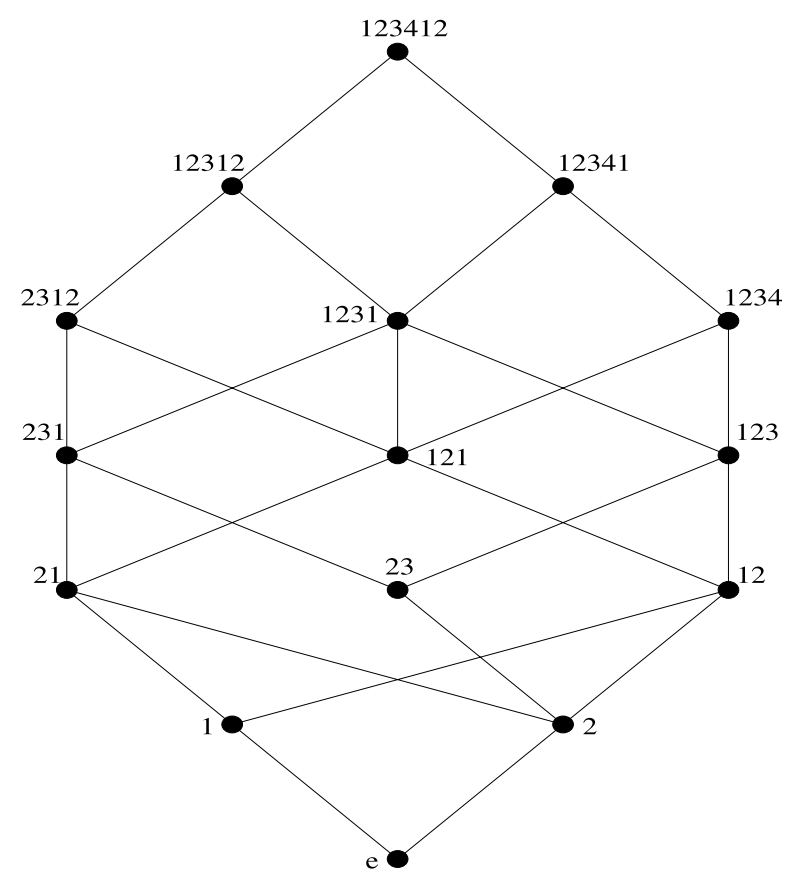

Fig. 1 A picture of the poset $\operatorname{Br}(\iota(\theta))$ when $W$ is the symmetric group $\mathfrak{S}_{6} \cong A_{5}$ and $\theta$ is the unique non-trivial automorphism (which sends the transposition $s_{i}=(i, i+1)$ to $\left.s_{6-i}\right)$. The vertex labels in the figure are index sequences of reduced $\underline{S}$-expressions for the corresponding twisted identities. For example, 2312 indicates the twisted identity $\underline{s}_{2} \underline{s}_{3} \underline{s}_{1} \underline{s}_{2}$.

Example 3.4 If $W$ is finite, it contains a unique longest element $w_{0}$. Then, $\theta(x)=$ $w_{0} x w_{0}$ defines an involutive automorphism of $(W, S)$. In this situation, $u \in \iota(\theta) \Leftrightarrow$ $u=w_{0} w^{-1} w_{0} w$ for some $w \in W$. This means that $u \in \iota(\theta)$ if and only if $w_{0} u$ and $w_{0}$ are conjugate. Now, (left or right) multiplication by $w_{0}$ is an antiautomorphism of $\operatorname{Br}(W)$. The poset $\operatorname{Br}(\iota(\theta))$ is therefore isomorphic to the dual of the subposet of $\operatorname{Br}(W)$ induced by the conjugacy class of the longest element.

In the special case when $W$ is the symmetric group $\mathfrak{S}_{2 n}$, the conjugacy class of $w_{0}$ consists of all fixed point free involutions of [2n]. Equivalently, if we think of a permutation in terms of its disjoint cycle decomposition, the conjugacy class of $w_{0}$ corresponds to the set of complete matchings on $2 n$ elements. Thus, $\operatorname{Br}(\iota(\theta))$ in this case gives (the dual of) a Bruhat order on matchings.

We mention in this context that the literature already contains a "Bruhat order" on matchings defined by Deodhar and Srinivasan [6]. Their poset is, however, strictly weaker than the dual of $\operatorname{Br}(\iota(\theta))$, although both posets are graded with the same rank function; see Remark 5.3.

\section{The Bruhat order on twisted identities}

We now turn to the core of the paper, namely the study of $\operatorname{Br}(\iota(\theta))$. To begin with, we observe that it sometimes coincides with the more familiar $\operatorname{Br}(\mathfrak{I}(\theta))$. 
Proposition 4.1 If there exists no $s \in S$ such that $\theta(s)$ and $s$ are conjugate, then $\iota(\theta)=\mathfrak{I}(\theta)$.

Proof Notice that $\theta(s) w s=w$ if and only if $w^{-1} \theta(s) w=s$. Thus, if $s$ and $\theta(s)$ are not conjugate, we have $w \underline{s}=\theta(s) w s$ for all $w$. Now, the assertion is a consequence of Proposition 2.5 and the remarks that follow it.

The hypothesis of Proposition 4.1 means that for all $s \in S$, every path from $s$ to $\theta(s)$ in the Coxeter graph of $W$ contains an edge with an even label; see [2, Exercise 1.16]. For example, Proposition 4.1 applies when $W=F_{4}$ with $\theta$ being the unique non-trivial automorphism.

We will find that $\iota(\theta)$ is particularly well-behaved if $\theta$ never flips an edge with an odd label in the Coxeter graph of $W$.

Definition 4.2 Say that $\theta$ has the no odd flip (NOF) property if $m(s, \theta(s))$ is even or infinite for all $s \neq \theta(s) \in S$.

For example, if $W$ is finite and irreducible, $\theta$ has the NOF property unless $W$ is of type $A_{2 n}$ or $I_{2}(2 n+1), n \in \mathbb{N}$, and $\theta$ is the unique non-trivial automorphism.

We now proceed to prove a series of lemmata that put restrictions on the behaviour of elements of twisted absolute length 1 when $\theta$ has the NOF property. Informally put, these restrictions ensure that intervals in $\operatorname{Br}(\iota(\theta))$ never can be "sparse enough" not to inherit the gradedness from $\operatorname{Br}(\mathfrak{I}(\theta))$.

Lemma 4.3 Suppose $W$ is irreducible and finite. If $\ell^{\theta}\left(w_{0}\right)=1$, then $W$ is either the rank one group $A_{1}$ or the dihedral group $I_{2}(m)$ for some odd integer $m$.

Proof If $\theta=\mathrm{id}$, the twisted absolute length coincides with the absolute length. Hence, $w_{0}$ is a reflection. It follows, for example by the classification of finite Coxeter groups, that $W=A_{1}$ or $W=I_{2}(m), m$ odd.

Now, assume $\theta \neq$ id. Obviously, $\ell^{\theta}(w)$ and $\ell(w)$ always have the same parity. In particular, $\ell\left(w_{0}\right)$ is odd. By inspection of Coxeter graphs, the only groups with $\ell\left(w_{0}\right)$ odd that admit a nontrivial $\theta$ are $I_{2}(m), m$ odd, and $A_{m}, m \equiv 1,2(\bmod 4)$. These groups have $\theta(w)=w_{0} w w_{0}$ as unique nontrivial automorphism; see [2, Exercise 4.10]. Therefore, $w \mapsto w_{0} w$ is a bijection which sends $\operatorname{Br}(\Im(\theta))$ to the dual of $\operatorname{Br}(\Im(i d))$. In particular, the top element $w_{0}$ has the same rank in $\operatorname{Br}(\Im(\theta))$ as in $\operatorname{Br}(\Im(\mathrm{Id}))$. This implies $\ell^{\prime}\left(w_{0}\right)=\ell^{\theta}\left(w_{0}\right)=1$, i.e. that $w_{0}$ is again a reflection. Hence, $W$ is the dihedral group $I_{2}(m)$ for some odd $m$.

Lemma 4.4 Let $W$ be finite and assume $\theta$ has the NOF property. If $\ell^{\theta}\left(w_{0}\right)=1$, then $w_{0}$ has a reduced $\underline{S}$-expression beginning with $\underline{s}$ for some $\theta$-fixed $s \in S$.

Proof The twisted absolute length clearly is additive over direct products of $\theta$ invariant Coxeter systems. Thus, $W$ decomposes as $W=W_{J} \times W_{S \backslash J}$ where $\ell^{\theta}\left(w_{J}\right)=1, \ell^{\theta}\left(w_{S \backslash J}\right)=0, W_{J}$ is irreducible and $J \subseteq S$ is invariant under $\theta$. Choose a reduced $\underline{S}$-expression $\underline{s}_{1} \cdots \underline{s}_{k}$ for $w_{S \backslash J}$. 
Lemma 4.3 shows that either $W_{J} \cong A_{1}$ or $W_{J} \cong I_{2}(m), m$ odd. In the former case, $w_{J}=\underline{s}$ for some $\theta$-fixed $s \in S$. This implies that $w_{0}$ has the reduced $\underline{S}$-expression $\underline{s s}_{1} \cdots \underline{s}_{k}$. In the latter situation, $w_{J}$ has the reduced $\underline{S}$-expression $\underline{s} \underline{s}^{\prime} \underline{s s^{\prime}} \cdots((m+1) / 2$ letters $)$, where $J=\left\{s, s^{\prime}\right\}$. The hypothesis on $m(s, \theta(s))$ means that $s$ and $s^{\prime}$ are $\theta$-fixed. Hence, $\underline{s s^{\prime}}{\underline{s s^{\prime}}}^{\prime} \underline{s}_{1} \cdots \underline{s}_{k}$ is a reduced expression for $w_{0}$ of the desired form.

Lemma 4.5 Suppose $\theta$ has the NOF property. Then, given $v \in \mathfrak{I}(\theta)$ with $\ell^{\theta}(v)=1$, $v$ covers at most one twisted identity in $\operatorname{Br}(\mathfrak{I}(\theta))$.

Proof By Lemma 2.6, $\ell^{\theta}(v)=1$ implies $v=w_{J} \underline{s}_{1} \cdots \underline{s}_{k}$ with $\ell(v)=\ell\left(w_{J}\right)+2 k$ for some $\theta$-invariant $J \subseteq S$ satisfying $\ell^{\theta}\left(w_{J}\right)=1$. Invoking Lemma 4.4, we may assume $J=\{s\}$. In other words, $v$ has a reduced $\underline{S}$-expression of the form $\underline{s}_{1} \cdots \underline{s}_{k}$, where $s$ is fixed by $\theta$.

Each reduced $k$-letter subword of $\underline{s s}_{1} \cdots \underline{s}_{k}$ except at most one begins with $\underline{s}$, hence represents an element of non-vanishing twisted absolute length. The exception is $\underline{s}_{1} \cdots \underline{s}_{k}$ (if this expression is reduced) which, by Theorem 2.8 , is the only candidate for a twisted identity covered by $v$.

Next, we state our main result on gradedness. As a notable special case, $\operatorname{Br}(\iota(\theta))$ is graded whenever the Coxeter graph of $W$ is a tree containing a $\theta$-fixed node. The nature of the rank function should not be surprising. Indeed, if $v=\underline{s}_{1} \cdots \underline{s}_{k}$ with $\rho(v)=k$, then $e<\underline{s}_{1}<\underline{s}_{1} \underline{s}_{2}<\cdots<\underline{s}_{1} \cdots \underline{s}_{k}$ is an unrefinable chain in $\operatorname{Br}(\iota(\theta))$. Hence, the rank function necessarily is $\rho$ whenever $\operatorname{Br}(\iota(\theta))$ is graded.

Theorem 4.6 If $\theta$ has the NOF property, then $\operatorname{Br}(\iota(\theta))$ is graded with rank function $\rho$.

Proof Since we know that $\operatorname{Br}(\Im(\theta))$ is graded with rank function $\rho$, it is enough to show that every interval $[u, v] \subseteq \operatorname{Br}(\iota(\theta))$ contains a chain of length $\rho(v)-\rho(u)$.

In order to get a contradiction, assume the theorem is false. Choose a minimal interval $[u, v] \subseteq \operatorname{Br}(\iota(\theta))$ which does not contain a chain of length $\rho(v)-\rho(u)$ and such that $\rho(v)$ is minimal among all such intervals.

Pick $s \in D_{R}(v)$. We must have $v \underline{s} \nsucceq u$; otherwise $[u, v]$ would not be minimal. By the lifting property, $u \underline{s}<u, v \underline{s}$. Minimality of $\rho(v)$ shows that $[u \underline{s}, v \underline{s}]$ contains some $a \in \iota(\theta)$ which covers $u \underline{s}$ in $\operatorname{Br}(\mathfrak{I}(\theta))$. Using the lifting property once again, we conclude that $a \underline{s}$ covers $u$ in $\operatorname{Br}(\mathfrak{I}(\theta))$. Minimality of $[u, v]$ implies $a \underline{s} \notin \iota(\theta)$. Thus, $a \underline{s}=a s$ and $\ell^{\theta}(a \underline{s})=\ell^{\theta}(a)+1=1$. Now, as covers two distinct twisted identities, namely $a$ and $u$. This contradicts Lemma 4.5 , and the proof is complete.

Unfortunately, Theorem 4.6 does not give the full picture. For example, one readily checks that with $W$ being any dihedral group and $\theta$ the non-trivial automorphism, $\operatorname{Br}(\iota(\theta))$ is graded, although this is not predicted by Theorem 4.6. At this point one may very well suspect that $\operatorname{Br}(\iota(\theta))$ is always graded. This is not true, as shown by the next example. 
Example 4.7 Suppose $W \cong \widetilde{A_{2}}$, the affine Weyl group corresponding to $A_{2}$. Its Coxeter generating set is $S=\left\{s_{1}, s_{2}, s_{3}\right\}$, and $m\left(s_{1}, s_{2}\right)=m\left(s_{1}, s_{3}\right)=m\left(s_{2}, s_{3}\right)=3$. Define the involution $\theta$ by $\theta\left(s_{1}\right)=s_{1}, \theta\left(s_{2}\right)=s_{3}$ and $\theta\left(s_{3}\right)=s_{2}$.

Consider the twisted identities $u=\underline{s}_{3}=s_{2} s_{3}$ and $v=\underline{s}_{2} \underline{s}_{1} \underline{s}_{3}=s_{2} s_{1} s_{3} s_{2} s_{1} s_{3}$. As an interval in $\operatorname{Br}(\Im(\theta))$, the open interval $(u, v)$ contains two elements, namely $\underline{s}_{2} \underline{s}_{3}=$ $s_{3} s_{2} s_{3}$ and $\underline{s}_{1} \underline{s}_{3}=s_{2} s_{1} s_{3}$, neither being a twisted identity. Thus, $e<u<v$ and $e<$ $\underline{s}_{2}<\underline{s}_{2} \underline{s}_{1}<v$ are unrefinable chains in $\operatorname{Br}(\iota(\theta))$. Hence, it is not graded.

Our attention is now turned to the topology of intervals in $\operatorname{Br}(\iota(\theta))$. The first results are valid without any restriction on $W$ or $\theta$.

Theorem 4.8 Consider an interval $[u, v] \subseteq \operatorname{Br}(\iota(\theta))$. If $u \underline{s}=u$ s for some $s \in D_{R}(v)$, then $(u, v)$ is collapsible.

Proof Let $I=(u, v)$. Our strategy is to invoke Theorem 2.1 after defining a complete acyclic matching $M$ on the face poset $P=P(\Delta(I))$.

Suppose $c$ is a chain in $I$, and define $x_{c}=\min \left\{x \in c \cup\{v\} \mid s \in D_{R}(x)\right\}$. Now, let $M: P \rightarrow P$ be given by

$$
M(c)= \begin{cases}c \cup\left\{x_{c} \underline{s}\right\} & \text { if } x_{c} \underline{s} \notin c, \\ c \backslash\left\{x_{c} \underline{s}\right\} & \text { otherwise. }\end{cases}
$$

Observe that $x_{c} \underline{s} \neq u$ because $u \underline{s}=u s \notin \iota(\theta)$. The lifting property ensures that $x_{c} \underline{s} \cup c$ is a chain in $I$. Since $s \notin D_{R}\left(x_{c} \underline{s}\right)$, we have $x_{c}=x_{M(c)}$ and therefore $M(M(c))=c$. Hence, $M$ is a complete matching.

To prove acyclicity of $M$, suppose we have four chains $c \prec M(c) \succ c^{\prime} \prec M\left(c^{\prime}\right)$, where $\prec$ is the covering relation in $P$ and $c \neq c^{\prime}$. By construction, $M(c)=c \cup\left\{x_{c} \underline{s}\right\}$ and $c^{\prime}=M(c) \backslash\{x\}$ for some $x \in M(c)$. In fact, $x=x_{c}=x_{M(c)}$; otherwise we would have $x_{c^{\prime}}=x_{c}$, implying $M\left(c^{\prime}\right)=c^{\prime} \backslash\left\{x_{c} \underline{s}\right\} \prec c^{\prime}$. We therefore conclude that $c^{\prime}=$ $\left\{x_{c} \underline{s}\right\} \cup c \backslash\left\{x_{c}\right\}$. In particular, $c^{\prime}$ has strictly fewer elements with $s$ as a descent than $c$ does. This shows the impossibility of a sequence

$$
c_{1} \prec M\left(c_{1}\right) \succ c_{2} \prec M\left(c_{2}\right) \succ \cdots \prec M\left(c_{t-1}\right) \succ c_{t}=c_{1}
$$

with $c_{1} \neq c_{2}$. In other words, $M$ is acyclic.

The strategy employed in the preceding proof is not quite applicable if $u \underline{s}=$ $\theta(s) u s, s \notin D_{R}(u)$, because chains that contain $u \underline{s}$ would not have a well-defined matching partner. Leaving these chains unmatched, however, allows us to conclude a result which is useful in recursive arguments.

Theorem 4.9 Consider an interval $[u, v] \subseteq \operatorname{Br}(\iota(\theta))$. If $u \underline{s}=\theta(s)$ us for some $s \in$ $D_{R}(v) \backslash D_{R}(u)$, then $(u, v)$ is homotopy equivalent to the suspension of $(u \underline{s}, v)$.

Proof Just as in the proof of Theorem 4.8, we define a matching on $P=P(\Delta(I))$, where $I=(u, v)$. This time, there will be critical cells. Define

$$
Z=\{c \in \Delta(I) \mid c \ni u \underline{s}\} .
$$


Now, arguing exactly as in the proof of Theorem 4.8 we have a complete acyclic matching $M: P \backslash Z \rightarrow P \backslash Z$.

The set of critical cells is the order filter $Z$ which consists of the cells in the open star of $u \underline{s}$. Corollary 2.2 therefore implies that $\Delta(I)$ is homotopy equivalent to the star of $u \underline{s}$ with the entire link of $u \underline{s}$ collapsed to a single point. The complex obtained in this way is homotopic to the suspension of the link of $u \underline{s}$, and this link is $\Delta((u \underline{s}, v))$.

Using Theorem 4.9, we can always compute the homotopy type of an interval in terms of that of another interval. The problem is that if $D_{R}(v) \subseteq D_{R}(u)$, we are only able to express $(u, v)$ in terms of larger intervals. This makes it difficult to set up an inductive argument since we may not reach a reasonable base case. To rectify the situation, we pay the price of restricting $W$ and $\theta$.

Let us call an interval $[u, v] \subseteq \operatorname{Br}(\iota(\theta))$ full if $\{w \in \mathfrak{I}(\theta) \backslash \iota(\theta) \mid u \leq w \leq v\}=\emptyset$.

Lemma 4.10 Suppose $\theta$ has the NOF property. If an interval $[u, v] \subseteq \operatorname{Br}(\iota(\theta))$ is full and $s \notin D_{R}(v) \cup D_{R}(u)$, then either $u \underline{s}=u s$ or $[u, v \underline{s}]$ is full (implying, in particular, $v \underline{s} \in \iota(\theta))$.

Proof Assume, in order to deduce a contradiction, that $u \underline{s}=\theta(s) u s$ and $[u, v \underline{s}]$ is not full. Choose $w \in[u, v]$ minimal with the property that $w \underline{s}=w s$; it exists since every element in $[u, v \underline{s}] \backslash[u, v]$ is of the form $w \underline{s}, w \in[u, v]$. We have $\ell^{\theta}(w s)=1$ and $u<w<v \underline{s}$.

Since $u<w<w s$ and intervals in $\operatorname{Br}(\mathfrak{I}(\theta))$ are PL spheres, $w s$ must cover some element $w^{\prime} \in \mathfrak{I}(\theta)$ with $u<w^{\prime} \neq w$. By the lifting property, $u \leq w^{\prime} \underline{s}<w$. Since $[u, v]$ is full, $w^{\prime} \underline{s} \in \iota(\theta)$. Minimality of $w$ now implies $w^{\prime} \in \iota(\theta)$. Hence, $w s$ covers more than one twisted identity, contradicting Lemma 4.5.

Lemma 4.11 Suppose $\theta$ satisfies the NOF property. Let $u<v$ for $u, v \in \iota(\theta)$ and suppose $v \underline{s}=v$ s for some $s \in S$. If $u \underline{s}=\theta(s) u s>u$, then $u \underline{s}<v$.

Proof We employ induction on $r=\rho(v)-\rho(u)$. The assertion is vacuously true if $r=1$, because in this case the hypotheses imply that $v s$ covers both $u \underline{s}$ and $v$, contradicting Lemma 4.5.

Now suppose $r>1$. Since $\operatorname{Br}(\iota(\theta))$ is graded, we can choose $w \in \iota(\theta)$ such that $w$ covers $u$ and $w<v$. If $u \underline{s}=w$, we are done. Otherwise, the lifting property implies that $w \underline{s}$ covers $u \underline{s}$. As in the $r=1$ case above, $w \underline{s}=w s$ is impossible, so $w \underline{s}=$ $\theta(s) w s$. By the induction assumption, $w \underline{s}<v$. Hence, $u \underline{s}<v$.

Theorem 4.12 Assume $\theta$ has the NOF property. Consider an interval $[u, v] \subseteq$ $\operatorname{Br}(\iota(\theta))$. If it is full, then $(u, v)$ is a PL sphere of dimension $\rho(v)-\rho(u)-2$. Otherwise, $(u, v)$ is $\mathbb{Z}$-acyclic.

Proof If $[u, v]$ is full, the assertion follows immediately from the corresponding result on $\operatorname{Br}(\Im(\theta))$, namely [10, Corollary 4.6]. 
Suppose $[u, v]$ is not full, and let $I=(u, v)$. Proceeding by induction on $\rho(v)$, we choose $s \in D_{R}(v)$. There are two cases depending on whether or not $s$ also is a descent of $u$.

Case I, $s \notin D_{R}(u)$.

If $u \underline{s}=u s$, Theorem 4.8 shows that $I$ is collapsible, in particular contractible and $\mathbb{Z}$-acyclic. By Lemma 4.10 , this is always the case if $[u, v s]$ is full. Therefore, we may assume that $[u, v \underline{s}]$ is not full and $u \underline{s}=\theta(s) u s$. By the induction assumption, $(u, v \underline{s})$ is $\mathbb{Z}$-acyclic.

Let $P=P(\Delta(I))$. Given a chain $c \in P$, define $x_{c}=\max \{x \in c \cup\{u\} \mid x \underline{s}=$ $\theta(s) x s>x\}$; this set is nonempty since it contains $u$. Let $Z=\{c \in P \mid v \underline{s} \in c\}=$ $\left\{c \in P \mid x_{c}=v \underline{s}\right\}$ and define $M: P \backslash Z \rightarrow P \backslash Z$ by

$$
M(c)= \begin{cases}c \cup\left\{x_{c} \underline{s}\right\} & \text { if } x_{c} \underline{s} \notin c, \\ c \backslash\left\{x_{c} \underline{s}\right\} & \text { if } x_{c} \underline{s} \in c .\end{cases}
$$

Lemma 4.11 together with the lifting property proves that $c \cup\left\{x_{c} \underline{s}\right\} \in P$. Observing that $x_{c}=x_{M(c)}$, we conclude that $M$ is a matching on $P$ with set of critical cells $Z$. An argument completely analogous to the acyclicity part of the proof of Theorem 4.8 shows that $M$ is an acyclic matching. Exactly as in the proof of Theorem 4.9, this implies that $I$ is homotopy equivalent to the suspension of the $\mathbb{Z}$-acyclic complex $(u, v \underline{s})$. Since a complex and its suspension have isomorphic reduced homology groups up to an index shift, $I$ is $\mathbb{Z}$-acyclic.

Case II, $s \in D_{R}(u)$.

By Lemma 4.10, $[u \underline{s}, v \underline{s}]$ is not full, and therefore $(u \underline{s}, v \underline{s})$ is $\mathbb{Z}$-acyclic. Combining Case I with Theorem 4.9, we have the following homotopy equivalences:

$$
\Sigma I \simeq(u \underline{s}, v) \simeq \Sigma(u \underline{s}, v \underline{s})
$$

where $\Sigma$ denotes suspension. Hence, the suspension of $I$ is $\mathbb{Z}$-acyclic. Therefore, $I$ is $\mathbb{Z}$-acyclic, too.

\section{The Poincaré series of $\iota(\theta)$}

From a combinatorial point of view, the Poincaré series of $W$ is simply the length generating function:

$$
\operatorname{Poin}(W ; t)=\sum_{w \in W} t^{\ell(w)} .
$$

Analogously, we may define the Poincaré series of $\iota(\theta)$ to be

$$
\operatorname{Poin}(\iota(\theta) ; t)=\sum_{w \in \iota(\theta)} t^{\rho(w)} .
$$

In particular, $\operatorname{Poin}(\iota(\theta) ; t)$ is the rank generating function for $\operatorname{Br}(\iota(\theta))$ whenever it is graded. 
Since $\iota(\theta)$ is, in some sense, a quotient of Coxeter groups (cf. Example 3.3), one may naively hope that its Poincaré series is a quotient of the series of the corresponding groups; let us say that $\operatorname{Poin}(\iota(\theta) ; t)$ factors through $\theta$ if $\operatorname{Poin}(W ; t)=$ $\operatorname{Poin}(\iota(\theta) ; t) \operatorname{Poin}(\operatorname{Fix}(\theta) ; t)$.

With $W=A_{2}$ and $\theta$ given by conjugation with the longest element, for example, one obtains $\operatorname{Poin}(\iota(\theta) ; t)=1+2 t$, whereas $\operatorname{Poin}(W ; t) / \operatorname{Poin}(\operatorname{Fix}(\theta) ; t)=1+$ $t+t^{2}$. Thus, in this case, Poin $(\iota(\theta) ; t)$ does not factor through $\theta$. Intriguingly, though, factorisation does occur in several situations, as we shall see below.

First, we give a necessary condition for the Poincaré series of $\iota(\theta)$ to factor through $\theta$. The condition is that $\theta$ (seen as a Coxeter graph automorphism) is not allowed to flip edges unless they are labelled $\infty$.

Proposition 5.1 If $\operatorname{Poin}(W ; t)=\operatorname{Poin}(\iota(\theta) ; t) \operatorname{Poin}(\operatorname{Fix}(\theta) ; t)$, then $m(s, \theta(s)) \in$ $\{1,2, \infty\}$ for all $s \in S$.

Proof Suppose equality holds, and consider the coefficient of the linear term on both sides. On the left hand side, this coefficient is $|S|$. The elements of length 1 in $\operatorname{Fix}(\theta)$ (i.e. the Coxeter generators) are the longest elements in the finite parabolic subgroups of the form $\langle s, \theta(s)\rangle$. In $\iota(\theta)$, the elements of rank 1 are the twisted identities of the form $\underline{s}, s \in S$. Now, $\underline{s}$ is a twisted identity if and only if $\theta(s) \neq s$. Moreover, for $s \neq s^{\prime}, \underline{s}=\underline{s}^{\prime}$ if and only if $\theta(s)=s^{\prime}$ and $m(s, \theta(s))=2$. As a consequence, the linear coefficient of the right hand side is

$|\{\{s, \theta(s)\} \mid m(s, \theta(s))<\infty\}|+|S|-|\{s \mid s=\theta(s)\}|-|\{\{s, \theta(s)\} \mid m(s, \theta(s))=2\}|$.

This is equal to $|S|$ if and only if $m(s, \theta(s)) \in\{1,2, \infty\}$ for all $s \in S$.

It turns out that in the finite setting, Proposition 5.1 actually gives a characterisation of the $(W, \theta)$ for which the factorisation phenomenon occurs. (The label $\infty$, of course, cannot occur in this case).

Theorem 5.2 Suppose $W$ is finite. Then, Poin $(\iota(\theta) ; t)$ factors through $\theta$ if and only if $m(s, \theta(s)) \in\{1,2\}$ for all $s \in S$, i.e. if and only if $s$ commutes with $\theta(s)$ for all $s \in S$.

Proof Suppose $J \subseteq S$ is minimal such that $\theta(J)=J$ and $m\left(s, s^{\prime}\right)=2$ whenever $s \in J, s^{\prime} \notin J$. Then, either $W_{J}$ is irreducible or $W_{J}$ is isomorphic to the direct product of two isomorphic irreducible Coxeter groups that are interchanged by $\theta$ as in Example 3.2. To prove the theorem it therefore suffices to check that it holds in the setting of Example 3.2 and for the finite irreducible groups that satisfy the hypotheses.

To begin with, return to the setup in Example 3.2. Observe that $\operatorname{Fix}(\theta) \cong W$. Furthermore, the rank of $\left(w, w^{-1}\right) \in \iota(\theta)$ is $\ell(w)$, so that $\operatorname{Poin}(\iota(\theta) ; t)=$ $\operatorname{Poin}(W ; t)$. Since Poin $(W \times W ; t)=\operatorname{Poin}(W ; t)^{2}$, we have $\operatorname{Poin}(W \times W ; t)=$ $\operatorname{Poin}(\iota(\theta) ; t) \operatorname{Poin}(\operatorname{Fix}(\theta) ; t)$.

It remains to consider the finite irreducible groups. If $\theta=\mathrm{id}$, the assertion is trivial. If not, the groups that satisfy the criteria are $A_{2 n-1} \cong \mathfrak{S}_{2 n}, D_{n}$ and $E_{6}$.

Type A. Consider the symmetric group case $W=\mathfrak{S}_{2 n}$ with $\theta$ given by conjugation with the longest element $w_{0}$ (the reverse permutation). Let us verify that $\operatorname{Poin}(\iota(\theta) ; t)$ factors through $\theta$. 
It is known [18, Supplementary problem 1.24] that the fixed point free involutions $F(2 n)$ in $\mathfrak{S}_{2 n}$ have the following generating function:

$$
I(n ; t)=\sum_{\pi \in F(2 n)} t^{\operatorname{inv}(\pi)}=t^{n} \prod_{i=0}^{n-1}\left(1+t^{2}+t^{4}+\cdots+t^{4 i}\right),
$$

where inv $(\pi)$ denotes the number of inversions in $\pi$, which is the same as the Coxeter length of $\pi$. Since $\iota(\theta)=w_{0} F(2 n)$ (Example 3.4), $\ell\left(w_{0} w\right)=2 n^{2}-n-\ell(w)$ for all $w \in \mathfrak{S}_{2 n}$ and $\rho(w)=\ell(w) / 2$ for all $w \in \iota(\theta)$, we obtain

$$
\begin{aligned}
\operatorname{Poin}(\iota(\theta) ; t) & =\left(t^{1 / 2}\right)^{2 n^{2}-n} I\left(n ; t^{-1 / 2}\right)=t^{n(n-1)} \prod_{i=0}^{n-1}\left(1+t^{-1}+t^{-2}+\cdots+t^{-2 i}\right) \\
& =\prod_{i=0}^{n-1}\left(1+t+t^{2}+\cdots+t^{2 i}\right) .
\end{aligned}
$$

It is well-known, and straightforward to check, that $\operatorname{Fix}(\theta) \cong B_{n}$, the hyperoctahedral group of rank $n$. Now,

$$
\operatorname{Poin}\left(\mathfrak{S}_{2 n} ; t\right)=\prod_{i=0}^{2 n-1}\left(1+t+t^{2}+\cdots+t^{i}\right)
$$

and

$$
\operatorname{Poin}\left(B_{n} ; t\right)=\prod_{i=1}^{n}\left(1+t+t^{2}+\cdots+t^{2 i-1}\right) .
$$

Thus, Poin $(W ; t)=\operatorname{Poin}(\iota(\theta) ; t) \operatorname{Poin}(\operatorname{Fix}(\theta) ; t)$ in this situation.

Type D. Let $W=D_{n}$ with the Coxeter generators being labelled as described in the Coxeter graph below.

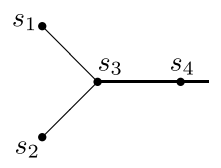

Define $\theta$ by $\theta\left(s_{1}\right)=s_{2}, \theta\left(s_{2}\right)=s_{1}$ and $\theta\left(s_{i}\right)=s_{i}$ for all $i>2$.

It is not hard to realise that $\iota(\theta)$ consists precisely of the elements of the form $\underline{s}_{2} \underline{s}_{3} \cdots \underline{s}_{k}$ for $k \in[n]$ (if $k=1$, we interpret this as the identity element $e$ ). In particular, Poin $(\iota(\theta) ; t)=1+t+t^{2}+\cdots+t^{n-1}$. Noting that $\operatorname{Fix}(\theta) \cong B_{n-1}$ (whose Poincaré series was presented in the type $A$ case above) and

$$
\operatorname{Poin}\left(D_{n} ; t\right)=\left(1+t+t^{2}+\cdots+t^{n-1}\right) \operatorname{Poin}\left(B_{n-1} ; t\right),
$$

we again conclude that $\operatorname{Poin}(\iota(\theta) ; t)$ factors through $\theta$.

Type E. When $\theta$ is the unique non-trivial automorphism of the Coxeter graph of $E_{6}$, the Hasse diagram of $\operatorname{Br}(\iota(\theta))$ is displayed in Figure 2. Inspecting it, one 


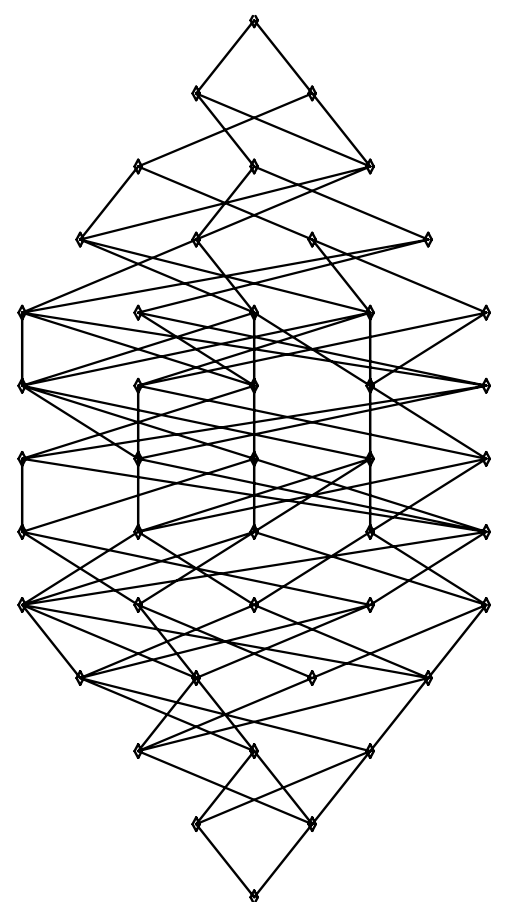

Fig. 2 The poset $\operatorname{Br}(\iota(\theta))$ when $W=E_{6}$ and $\theta$ is the non-trivial automorphism.

obtains the rank generating function and verifies that $\operatorname{Poin}\left(E_{6} ; t\right)$ factors through $\theta$. Here, $\operatorname{Fix}(\theta) \cong F_{4}$.

Remark 5.3 Return to the type A case $W=\mathfrak{S}_{2 n}$ with $\theta$ given by conjugation with $w_{0}$. Then, Poin $(\iota(\theta) ; t)$ coincides with the rank generating function for Deodhar and Srinivasan's "Bruhat order" on $F(2 n)$ [6, Theorem 1.3(i)] which was mentioned in Example 3.4. In fact, although formulated differently in [6], the definition of their rank function wt : $F(2 n) \rightarrow \mathbb{N}$ is readily seen to yield

$$
\operatorname{wt}(\sigma)=\frac{\operatorname{inv}(\sigma)-n}{2}=n(n-1)-\operatorname{inv}\left(w_{0} \sigma\right) / 2=n(n-1)-\rho\left(w_{0} \sigma\right),
$$

so that

$$
\sum_{\sigma \in F(2 n)} t^{\mathrm{wt}(\sigma)}=t^{n(n-1)} \operatorname{Poin}(\iota(\theta) ; 1 / t)=\operatorname{Poin}(\iota(\theta) ; t),
$$

where the last equality is valid since $\operatorname{Poin}(\iota(\theta) ; t)$ is a symmetric polynomial in this case. 


\section{Further comments and open questions}

\subsection{Gradedness}

We have already commented on the fact that, although Theorem 4.6 does not predict it in all cases, $\operatorname{Br}(\iota(\theta))$ is graded whenever $W$ is dihedral. In fact, the only instances of non-graded $\operatorname{Br}(\iota(\theta))$ that we know of follow from Example 4.7 and simple variations thereof ${ }^{3}$. Ultimately, of course, we would like a complete characterisation of the $(W, S)$ and $\theta$ for which $\operatorname{Br}(\iota(\theta))$ is graded. A step in this direction would be to resolve the following two problems.

Conjecture 6.1 When $W=A_{2 m} \cong \mathfrak{S}_{2 m+1}, \operatorname{Br}(\iota(\theta))$ is always graded.

Aided by Stembridge's Maple packages coxeter and posets [20,21], we have verified Conjecture 6.1 for $m \leq 4$. The conjecture would follow from an affirmative answer to

Question 6.2 Is $\operatorname{Br}(\iota(\theta))$ graded whenever the Coxeter graph of $W$ is a tree?

\subsection{Interval topology}

Consider the setup of Theorem 4.12. By a result of Whitehead [22], a collapsible PL manifold is a PL ball (see e.g. [15, Corollary 3.28]). By induction on the rank, we may assume that the proper subintervals of $(u, v)$ are PL spheres or balls, hence that $(u, v)$ is a PL manifold. Thus, if we were somehow able to replace " $\mathbb{Z}$-acyclic" by "collapsible" in the conclusion of Theorem 4.12, we would have a proof of the next conjecture.

Conjecture 6.3 Suppose $\theta$ has the NOF property. Consider an interval $[u, v] \subseteq$ $\operatorname{Br}(\iota(\theta))$. If it is full, then $(u, v)$ is a PL sphere of dimension $\rho(v)-\rho(u)-2$. Otherwise, $(u, v)$ is a PL ball of the same dimension.

The conditions on $(W, S)$ and $\theta$ which guarantee that $\operatorname{Br}(\iota(\theta))$ is graded in Theorem 4.6 are exactly those for which Theorem 4.12 asserts that the intervals are PL spheres or $\mathbb{Z}$-acyclic (namely, $\theta$ should satisfy the NOF property). This immediately leads to the next question.

Question 6.4 Does there exist a graded interval in some $\operatorname{Br}(\iota(\theta))$ which is neither a PL sphere nor $\mathbb{Z}$-acyclic?

If Conjecture 6.3 is valid, one would of course like to replace " $\mathbb{Z}$-acyclic" by "ball" in Question 6.4.

Like any non-graded interval, the one in Example 4.7 is neither a PL ball nor sphere. Note, however, that it is homotopy equivalent to a 0 -dimensional sphere.

\footnotetext{
${ }^{3}$ For example, the argument is not affected if $m\left(s_{1}, s_{2}\right)=m\left(s_{1}, s_{3}\right)$ is increased. Similarly, $m\left(s_{2}, s_{3}\right)$ can be increased to any odd number $\geq 3$ with simple adjustments.
} 
Question 6.5 Are there intervals in some $\operatorname{Br}(\iota(\theta))$ that are neither homotopy equivalent to spheres nor contractible?

The Möbius function of an interval coincides with the reduced Euler characteristic of its order complex. Thus, a negative answer to Question 6.5 would imply a negative answer to the following:

Question 6.6 Is there some $\operatorname{Br}(\iota(\theta))$ whose Möbius function takes values outside $\{-1,0,1\}$ ?

When $W=A_{2 m}, m \leq 4$, we have used [20, 21] to verify that the range of the Möbius function of $\operatorname{Br}(\iota(\theta))$ is $\{-1,0,1\}$.

\subsection{Directedness}

Whenever $W$ is finite, $\operatorname{Br}(\mathfrak{I}(\theta))$ and $\operatorname{Br}(W)$ contain a unique top element $w_{0}$. This element is, however, usually not a twisted identity. Indeed $\operatorname{Br}(\iota(\theta))$ does not always possess a unique maximal element.

Proposition 6.7 If $W$ is irreducible and finite, then $\operatorname{Br}(\iota(\theta))$ has more than one maximal element if and only if $W$ is of type $A_{2 m}$ or $I_{2}(2 m+1)$, for some positive integer $m$, and $\theta$ is the unique non-trivial automorphism.

Proof If $\theta=\mathrm{id}, e$ is the only twisted identity. Consulting the classification of finite irreducible Coxeter groups, we find that the groups that admit a nontrivial $\theta$ are $A_{n}$, $D_{n}, E_{6}, F_{4}$ and $I_{2}(n)$. Furthermore, this $\theta$ is unique in all cases (except $D_{4}$, but the various choices are then equivalent). The dihedral groups $I_{2}(n)$ are easy to handle. Types $D$ and $E$ are covered by Theorem 5.2 whereas the type $F$ assertion follows from Proposition 4.1. It remains to understand type $A_{n}$. Here, one may study the Bruhat order on the conjugacy class of $w_{0}$ as described in Example 3.4. It is straightforward to check that the minimal elements in this class are the possible products of $\lceil n / 2\rceil$ mutually commuting Coxeter generators. If $n$ is odd, there is only one such product; otherwise there are $n / 2+1$ of them.

The property of having a unique maximal element has a counterpart in infinite groups. A poset is directed if every pair of elements has a common upper bound.

Question 6.8 For which $W, \theta$ is $\operatorname{Br}(\iota(\theta))$ a directed poset?

We proceed to mention some reasonably straightforward partial answers to Question 6.8.

It is known that $\operatorname{Br}(W)$ always is directed. A proof using the lifting property of $\operatorname{Br}(W)$ is given in [2]. Employing instead the lifting property of $\operatorname{Br}(\mathfrak{I}(\theta))$, it follows in exactly the same way that $\operatorname{Br}(\Im(\theta))$ is directed, too. Thus, in addition to the cases provided by Proposition 6.7, we may immediately conclude that $\operatorname{Br}(\iota(\theta))$ is directed whenever the hypothesis of Proposition 4.1 is satisfied.

Here is another situation in which directedness is relatively effortless to establish: 
Proposition 6.9 Assume $W$ is infinite. Suppose there is a partition $S=S_{1} \uplus S_{2} \uplus S_{3}$ with $\theta\left(S_{1}\right)=S_{2}$ and $S_{3}=\{s \in S \mid \theta(s)=s\}$ such that the elements of $S_{i}$ commute pairwise for $i=1,2,3$. Then, $\operatorname{Br}(\iota(\theta))$ is directed.

Proof Recall that a Coxeter element is a product of the Coxeter generators (in any order). Speyer [16] has shown that $\ell\left(c^{k}\right)=|S| k$ for all $k \in \mathbb{N}$ and an arbitrary Coxeter element $c$ whenever $W$ is infinite. Therefore, given $v, w \in W$, a large enough power of any Coxeter element is an upper bound for $v$ and $w$ in $\operatorname{Br}(W)$. Observe that $c=w_{S_{1}} w_{S_{3}} w_{S_{2}}$ is a Coxeter element in $W$. Moreover, $c^{2 k}=\theta\left(c^{-k}\right) c^{k} \in \iota(\theta)$. Thus, $\operatorname{Br}(\iota(\theta))$ is directed.

\subsection{The Poincaré series}

Proposition 5.1 and Theorem 5.2 immediately lead to the next question.

Question 6.10 Does Poin $(\iota(\theta) ; t)$ factor through $\theta$ if and only if $m(s, \theta(s)) \in$ $\{1,2, \infty\}$ for all $s \in S$ ?

In Section 5, in order to answer the finite case version of Question 6.10 affirmatively, we resorted to a case by case computation which did not shed much light on the situation. A combinatorial proof, type independent if possible, would be much preferred. Specifically, what we are looking for is this:

Problem 6.11 Given that Poin $(\iota(\theta) ; t)$ factors through $\theta$, construct a bijection $\phi$ : $\iota(\theta) \times \operatorname{Fix}(\theta) \rightarrow W$ such that $\ell(\phi((w, f)))=\rho(w)+\ell_{\theta}(f)$, where $\ell_{\theta}$ denotes the length of $f$ in terms of the canonical Coxeter generators of $\operatorname{Fix}(\theta)$ (cf. Example 3.3).

Recalling from Example 3.3 that we may identify $\iota(\theta)$ with the set of cosets $\operatorname{Fix}(\theta) \backslash W$, there is of course a natural bijection $\iota(\theta) \times \operatorname{Fix}(\theta) \rightarrow W$ defined by sending $(\operatorname{Fix}(\theta) w, f)$ to $f w$, but in general it does not have the desired properties.

\section{References}

1. Björner, A.: Topological methods. In: Graham, R., Grötschel, M., Lovász, L. (eds.) Handbook of Combinatorics, pp. 1819-1872. North-Holland, Amsterdam (1995)

2. Björner, A., Brenti, F.: Combinatorics of Coxeter Groups. Graduate Texts in Mathematics, vol. 231. Springer, New York (2005)

3. Björner, A., Wachs, M.: Bruhat order of Coxeter groups and shellability. Adv. Math. 43, 87-100 (1982)

4. Chari, M.: On discrete Morse functions and combinatorial decompositions. Discrete Math. 217, 101113 (2000)

5. Deodhar, V.V.: Some characterizations of Bruhat ordering on a Coxeter group and determination of the relative Möbius function. Invent. Math. 39, 187-198 (1977)

6. Deodhar, R.S., Srinivasan, M.K.: A statistic on involutions. J. Algebr. Comb. 13, 187-198 (2001)

7. Forman, R.: Morse theory for cell complexes. Adv. Math. 134, 90-145 (1998)

8. Hée, J.-Y.: Systèmes de racines sur un anneau commutatif totalement ordonné. Geom. Dedicata 37, 65-102 (1991)

9. Hultman, A.: Fixed points of involutive automorphisms of the Bruhat order. Adv. Math. 195, 283-296 (2005) 
10. Hultman, A.: The combinatorics of twisted involutions in Coxeter groups. Trans. Am. Math. Soc. 359, 2787-2798 (2007)

11. Humphreys, J.E.: Reflection Groups and Coxeter Groups. Cambridge Studies in Advanced Mathematics, vol. 29. Cambridge Univ. Press, Cambridge (1990)

12. Mühlherr, B.: Coxeter groups in Coxeter groups. In: Finite Geometry and Combinatorics, Deinze, 1992. London Math. Soc. Lecture Note Ser., vol. 191, pp. 277-287. Cambridge Univ. Press, Cambridge (1993)

13. Richardson, R.W., Springer, T.A.: The Bruhat order on symmetric varieties. Geom. Dedicata 35, 389436 (1990)

14. Richardson, R.W., Springer, T.A.: Complements to: The Bruhat order on symmetric varieties. Geom. Dedicata 49, 231-238 (1994)

15. Rourke, C.P., Sanderson, B.J.: Introduction to Piecewise-Linear Topology. Reprint, Springer Study Edition, Springer, Berlin (1982)

16. Speyer, D.E.: Powers of Coxeter elements in infinite groups are reduced, arXiv:0710.3188

17. Springer, T.A.: Some results on algebraic groups with involutions. Adv. Studies Pure Math. 6, 525$543(1985)$

18. Stanley, R.P.: Enumerative Combinatorics, vol. I, 2nd edn. Cambridge Univ. Press, Cambridge (1997)

19. Steinberg, R.: Endomorphisms of linear algebraic groups. Mem. Am. Math. Soc. 80, 1-108 (1968)

20. Stembridge, J.R.: coxeter ver. 2.4, Maple package, 2004, available from http://www.math.lsa. umich.edu/ jrs/maple.html

21. Stembridge, J.R.: posets ver. 2.3, Maple package, 2006, available from http://www.math.lsa.umich. edu/ jrs/maple.html

22. Whitehead, J.H.C.: Simplicial spaces, nuclei and $m$-groups. Proc. Lond. Math. Soc. 45, 243-327 (1939) 